\title{
Hydrogel-integrated plasmonic nanostructures on optical fiber facet for remote and real-time $\mathrm{pH}$ sensing
}

ShiJie Li, Wen-Di Li

ShiJie Li, Wen-Di Li, "Hydrogel-integrated plasmonic nanostructures on optical fiber facet for remote and real-time pH sensing," Proc. SPIE 10353, Optical Sensing, Imaging, and Photon Counting: Nanostructured Devices and Applications 2017, 103530V (29 August 2017); doi: 10.1117/12.2272550

Event: SPIE Nanoscience + Engineering, 2017, San Diego, California, United States 


\title{
Hydrogel-integrated plasmonic nanostructures on optical fiber facet for remote and real-time $\mathrm{pH}$ sensing
}

\author{
ShiJie $\mathrm{Li}^{\mathrm{a}}{ }^{\mathrm{a}}$, Wen-Di Li ${ }^{\mathrm{a}, \mathrm{b} *}$ \\ ${ }^{a}$ Department of Mechanical Engineering, the University of Hong Kong, Pokfulam, Hong Kong; \\ ${ }^{b}$ HKU-Zhejiang Institute of Research and Innovation (HKU-ZIRI), Hangzhou, Zhejiang 311300, \\ China
}

\begin{abstract}
In this work, we use ultraviolet nanoimprint lithography (UV-NIL) to transfer metallic nanostructures from a polymer mold to the facet of the optical fiber with $200 \mu \mathrm{m}$ core diameter. Once a polymer mold carrying nanopillar array is fabricated by thermal embossing, a thin layer of gold is deposited on it by thermal evaporation. Then the metallic nanostructure is transferred onto fiber facet by the cross-linked UV-cured resist. The transferred metallic nanostructures feature closely spaced double layers of disks and holes. Strong coupling between the metal disk and hole generates resonantly enhanced local electrical field under incident excitation light, as revealed by peaks and dips in the reflection spectra. A layer of hydrogel is coated and cross-linked on fiber facet as a $\mathrm{pH}$-sensing element. Hydrogel shrinks in acid and swells in basic solutions by containing different amount of water and thus has a different refractive index, which can be detected from the resonant reflection peaks/dips of plasmonic fiber probe. Our hydrogel fiber probe shows obvious spectrum response to solutions with $\mathrm{pH}$ values ranging from 1 to 8 . Under cycling test, the sensor remains stable for three cycles when switching between acid and basic solutions.
\end{abstract}

Keywords: Surface plasmon resonance, Fiber-optics sensor, Refractive index, Hydrogel, pH sensing, Nanoimprint

\section{INTRODUCTION}

Surface plasmon resonance (SPR) on periodic subwavelength metallic structures has drawn much attention on sensing applications because of its strong sensitivity to surrounding refractive index changes. ${ }^{1,2}$ There are increasing plasmonic sensors developed for environment monitoring ${ }^{3}$, hazardous gas detection ${ }^{4}$, food safety testing ${ }^{5}$ and disease diagnosis ${ }^{6}$. Integrating SPR nanostructure on optical fiber can realize more accessible and versatile plasmonic sensing applications especially in hazardous, remote and spatially limiting working space. ${ }^{7,8}$ Among many chemical sensing applications, new sensors for $\mathrm{pH}$ value measurement remain to be of wide interest, especially for minimally invasive measuring need. ${ }^{7}$

The optical fiber has merits to be a miniaturized $\mathrm{pH}$ sensor owing to its small cross-section and long working distance. Moreover, fiber-optic $\mathrm{pH}$ sensor takes advantages for its biocompatibility and absence of passing electrical signals. ${ }^{9}$ Fiber-optic $\mathrm{pH}$ sensors usually use functional sensing materials on fiber sidewall to perform $\mathrm{pH}$ sensing functions. ${ }^{10,11}$ However, this kind of fiber probes are brittle and not miniature in the longitudinal direction. These problems can be resolved when functional layer is patterned on the facet of optical fiber. Nanoimprint lithography (NIL) is proved to have the ability to pattern on fiber facet ${ }^{12}$, with quick speed and high-resolution nanostructures. Furthermore, fiber-optics has been investigated to diagnose stomach disorder and Gastroesophageal reflux ${ }^{13}$. The $\mathrm{pH}$-stimuli hydrogel, responding to a large range of $\mathrm{pH}$ values and having excellent biocompatibility, can be integrated on optical fiber for in vivo detection. ${ }^{14}$

In this work, we develop a new type of fiber-optic $\mathrm{pH}$ sensors featuring plasmonic nanostructures integrated with $\mathrm{pH}$ sensitive hydrogel coating, all fabricated on fiber facet by NIL. The plasmonic metallic nanostructure fabricated on the fiber facet is demonstrated with the capability of detecting local refractive index (RI) change when tested using a series of solutions with different RI. The pH-responsive hydrogel, coated and integrated with the plasmonic sensing structures, is responsible to change the local $\mathrm{RI}$ according to the $\mathrm{pH}$ value change, which varies the amount of water absorption in the hydrogel. The probe sensing capacity is also discussed in terms of cycling test and broad range $\mathrm{pH}$ values test. The hydrogel-integrated plasmonic fiber probe shows merits in rapid fabrication, remote working, and reversible $\mathrm{pH}$ detection.

*liwd@hku.hk

Optical Sensing, Imaging, and Photon Counting: Nanostructured Devices and Applications 2017, edited by

Manijeh Razeghi, Oleg Mitrofanov, José Luis Pau Vizcaíno, Chee Hing Tan, Proc. of SPIE Vol. 10353,

$103530 \mathrm{~V} \cdot$ C $2017 \mathrm{SPIE} \cdot \mathrm{CCC}$ code: $0277-786 \mathrm{X} / 17 / \$ 18 \cdot$ doi: $10.1117 / 12.2272550$ 


\section{EXPERIMENTAL SECTION}

The fabrication process for plasmonic optical fiber probe is presented in Figure 1. A 200- $\mu$ m core-diameter optical fiber with a bare fiber tip is prepared after striping and cleaving procedures. The flat fiber facet is achieved by standard polishing and grinding process, except for conventional epoxy used to fix fiber inside ferrule is replaced by rosin, which is brittle at room temperature but melts when heated to a certain temperature. Use of rosin ensures controllable polishing process to get a flat fiber facet. Then the fiber facet is extruded out of ferrule with $1 \mathrm{~cm}$ length, as shown in Figure 1a, during rosin heating to melting state by a butane lighter (Bic, France). A thin layer of UV-curable adhesive (NOA 61, Norland Products, Inc.) is then coated onto the extruded fiber facet from a carrying silicon substrate, illustrated in Figure 1b. Then the fiber facet is controlled to imprint on a gold-coated polymer (COC, TOPAS Advanced Polymers) mold with reverse nanostructures, as shown in Figure 1c. UV light is guided in fiber from its far end to cure adhesive between fiber facet and nanostructured mold. After the photoresist has been cured, the fiber tip is separated from the polymer template to get the plasmonic fiber probe. As a result, the cured adhesive layer presents nanohole array at its surface, fixing gold disk-array at its bottom and gold hole-array at its top surface, as illustrated in Figure 1e.

The front view SEM image reveals the morphology of nanofabricated fiber facet in Figure 2d. All the fiber handling processes are carefully controlled by a custom-built 3D platform integrated with an illumination source, mechanical stages, and monitoring cameras. NOA 61 adhesive has best chemical bond with metal and glass, and low adhesion with plastic. This property contributes to the easy separation of the gold layer from its polymer mold. The periodic nanostructures on COC mold are initially patterned on a COC flat film in a thermal-NIL equipment (YPL-NIL-SI400, China). A $1 \times 1 \mathrm{~cm}^{2}$ silicon hole-array mold is pressed onto the flat COC film surface with $0.4 \mathrm{MPa}$ pressure and $120^{\circ} \mathrm{C}$ heating temperature, for $15 \mathrm{~min}$. Therefore a pattern complementary to that on the silicon mold is created on the COC film surface after cooling process. Subsequently, the COC mold is released from the silicon mold and is placed in thermal evaporator and $40 \mathrm{~nm}$ thick gold is deposited on its nanostructured surface.

(a)

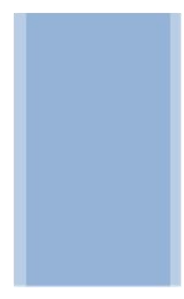

Flat fiber facet UV curable adhesive (b)

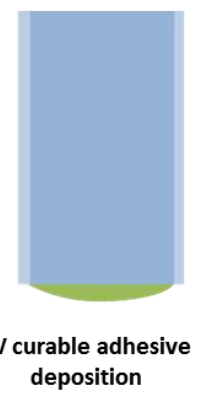

(c)

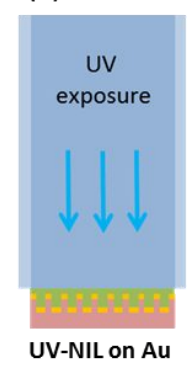

(d)

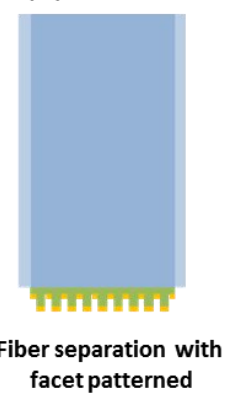

(e)

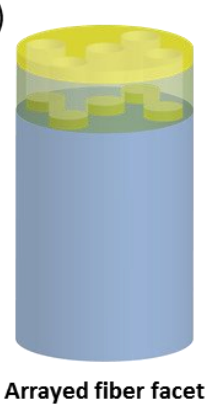

Figure 1. Schematics showing the UV-NIL process to fabricate arrayed fiber facet. (a) Bare and flat fiber facet after cleaving and polishing process. (b) A small droplet of UV-curable adhesive is coated on fiber facet. (c) UV-NIL process that fiber facet is pressed on Au coated COC mold, under UV exposure guided from same fiber. (d) Double gold layers transferring on fiber facet after releasing from COC mold. (e) 3D diagram of arrayed fiber facet showing hole-array and disk-array in (d).

For the preparation of pH-responsive hydrogel, 11.786g hydroxyethyl methacrylate (HEMA) and $0.237 \mathrm{~g}$ ethylene glycol dimethacrylate (EGDMA) are weighted and dissolve in DI water. The polymerization medium is then mixed with $1.1075 \mathrm{~g}$ acrylic acid (AA), $0.5034 \mathrm{~g}$ Brij58 and $0.195 \mathrm{~g}$ DMPA in $1.501 \mathrm{~mL}$ DI water and stirring by magnetic rods until the hydrogel precursor is clear. DMPA is photo-initiator and catalyzes hydrogel crosslinking process under UV exposure. Brij58 surfactant improves adhesion between the hydrogel and optical fiber facet. After that, we degas hydrogel precursor under 30 torr vacuum for 10 mins. The precursor is kept in a refrigerator at $4^{\circ} \mathrm{C}$.

Crosslinking of the catalyzed solution is initiated upon exposure to $365 \mathrm{~nm}$ UV light. The crosslinking procedure is done after 100 seconds UV exposure with $10 \mathrm{~mW} / \mathrm{cm}^{2}$ light power. A dip coating method is applied to integrate hydrogel on arrayed fiber probe. A small droplet of hydrogel precursor is attached on the arrayed fiber facet, after a short dip and liftoff process. An approximately $20 \mathrm{~mJ}$ UV illumination energy is then exposed on the hydrogel droplet guided through the fiber itself. Considering the fiber facet that the closer distance from fiber facet, the more curing energy the hydrogel receives, another flux UV exposure is applied from the front side of fiber facet for $1 \mathrm{~min}$. In this way, the HEMA hydrogel is successfully coated on plasmonic fiber probe as shown in Figure 2c, with quick synthesis speed and controllable hydrogel size. Following the cross-linking process, the hydrogel-coated plasmonic fiber probe is dipped in 
DI water for $1 \mathrm{~h}$, so that the hydrogel absorbs water to reach equilibrium volume. A SEM image shows HEMA hydrogel coated plasmonic fiber facet, proving that hydrogel successfully covers whole functional part of arrayed fiber facet (Figure 2e).

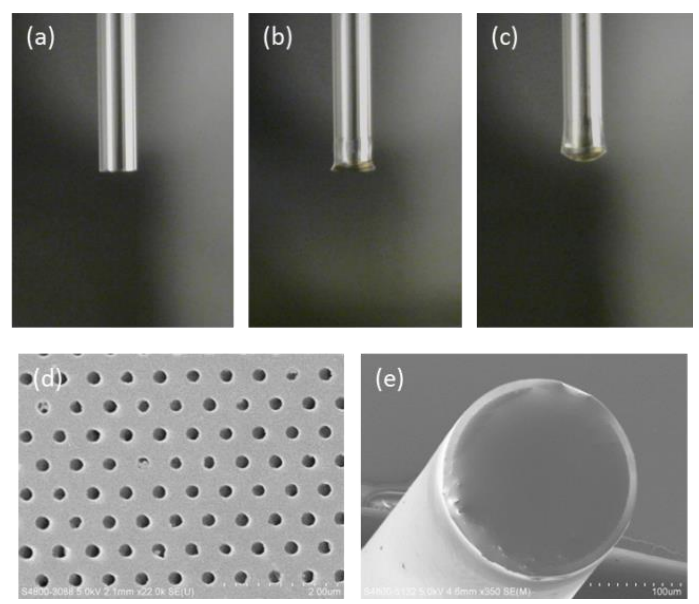

Figure 2. (a) Optical microscopy $(\mathrm{OM})$ image showing Bare and flat fiber facet after cleaving and polishing process. (b) OM image showing arrayed fiber facet as illustrated in Figure 1d and e. (c) HEMA hydrogel cross-linking on arrayed fiber facet. (d) SEM image showing morphology of arrayed fiber facet in (b). (e) SEM image showing HEMA hydrogel covering on arrayed fiber facet.

\section{RESULTS AND DISCUSSION}

The optical measurement for the plasmonic fiber probe is first conducted without hydrogel integration. The far end of arrayed fiber probe is connected to a halogen light source (HL-2000, Ocean Optics) and a spectrometer (HR2000+CGUV-VIS, Ocean Optics) through a bifurcated fiber, illustrated in Figure 3a. The reflection spectrum of the fiber-optic plasmonic probe is then recorded by the spectrometer in real-time. The sample reflectance is normalized by using a fiberoptic reference mirror, fabricated by depositing a $200 \mathrm{~nm}$-thick silver film on the facet of $200 \mu \mathrm{m}$ diameter multi-mode fiber. The plasmonic fiber tip is dipped into a series of solutions of standard RI liquids (Cargille Company), with the RI varying from 1.31 to 1.43 with $0.03 \mathrm{RI}$ step. After each immersion in the RI liquid, the fiber probe is rinsed with isopropanol alcohol (IPA) to remove residual RI liquid. As depicted in Figure 3b, the SPR dip in reflection spectrum redshifts with increasing RI. A fast Fourier transform (FFT) filter is applied to the original spectra to prevent random highfrequency intensity noise and spectral ripples. A straight dashed line in Figure $3 \mathrm{~b}$ indicates a linear correlation between spectral SPR wavelength and detected RI value. The arrayed fiber facet probe is capable of real-time detection of RI variations. HEMA hydrogel swells and shrinks in alkaline and acid aqueous solutions, respectively. ${ }^{15}$ Hydrogel RI fluctuates during the water absorption or desorption procedure and can be detected by the arrayed fiber facet. In this way, a $\mathrm{pH}$-responsive fiber probe is obtained.

(a)

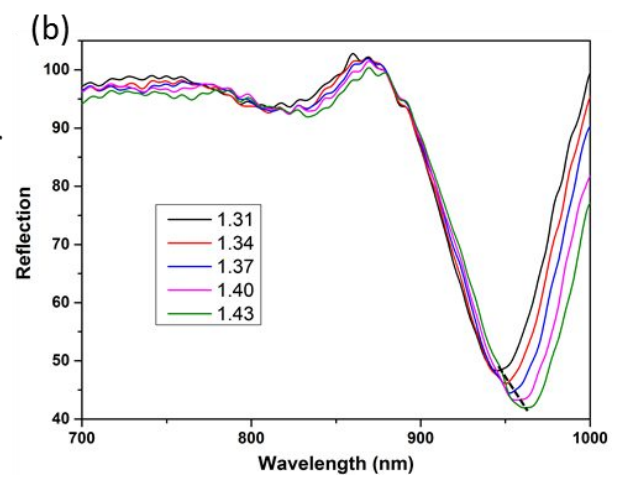

Figure 3. (a) Optical setup for the plasmonic fiber probe performing detection of aqueous solutions. (b) Measured reflection spectra for the fiber sensor in a series of refractive index liquid 
Phosphate-acetate buffered solutions are used as the analyte solutions to expose the hydrogel to a variety of $\mathrm{pH}$ values spanning from acid $(\mathrm{pH}=2.06)$ to alkaline $(\mathrm{pH}=8.06)$. To remove any effects of ionic strength from the hydrogel swelling, all solutions are adjusted to an ionic strength of $0.2 \mathrm{M}$ using $\mathrm{NaCl}$. To demonstrate that the spectral response for different $\mathrm{pH}$ solutions are directly related to hydrogel equilibrium states, the bare arrayed fiber probe (Figure $2 \mathrm{~b}$ ) is first inserted into acid and alkaline solutions. Minor spectral difference is observed in Figure 4a, and the small spectra shift corresponds to RI response of NOA 61 adhesive in different $\mathrm{pH}$ environment. When hydrogel integrated fiber probe is dipped into same acid and alkaline solutions, two types of spectral responses are shown in Figure $4 \mathrm{~b}$. These responses are over the entire visible-NIR region and are significantly stronger than responses from a probe with no hydrogel integrated. The marked changes are associated with plasmonic interactions with the pH-responsive dynamics of the hydrogel layer. The hydrogel swells when exposed in higher $\mathrm{pH}$ environment, and the absorbed water (RI=1.333) reduces the overall RI of the hydrogel. Therefore a blue shift is observed in Figure 4b, corresponding to an RI decrease when $\mathrm{pH}$ changes from 2.06 to 8.05 .

A series of measurements on the SPR dip wavelengths (as referenced to an initial reflection spectrum at time $=0$ ) demonstrate both the reversibility and stability of hydrogel-driven plasmonic responses (Figure 4c). No changes are observed for the SPR wavelength while a constant $\mathrm{pH}=2.06$ analyte solution continuously flows for 15 mins. Switching the solution $\mathrm{pH}$ to 8.05 for another 15 mins results SPR wavelength shift being measured and the result is stable when hydrogel reaches its equilibrium state. The results also present a faster response of HEMA hydrogel in lower $\mathrm{pH}$ value liquid, at which spectrum takes around $100 \mathrm{~s}$ to be stable, much shorter duration than $400 \mathrm{~s}$ when switching to higher $\mathrm{pH}$ value liquid. The data in Figure $4 \mathrm{c}$ also illustrates good reversibility of the fiber $\mathrm{pH}$ probe using representative response curves taken from a sequence of three complete $\mathrm{pH}$ switching cycles.
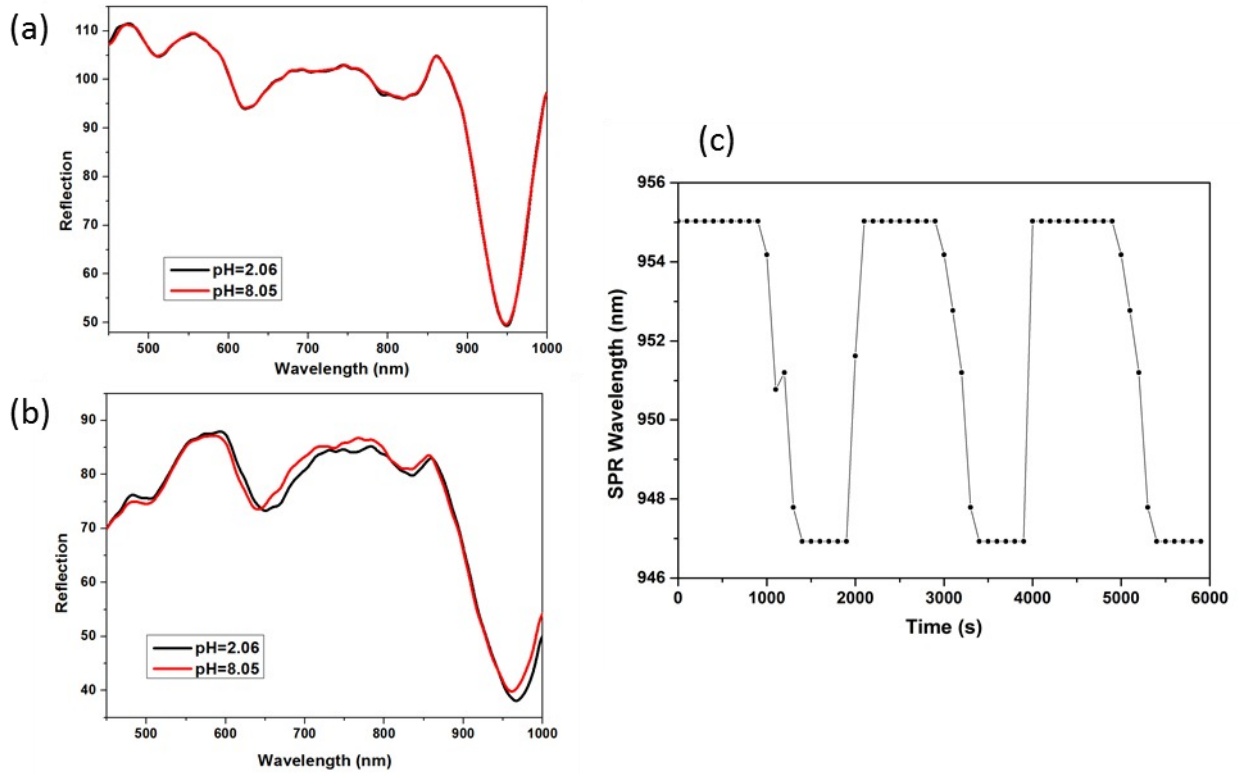

Figure 4. Reflection spectra of a plasmonic fiber probe in an acidic $\mathrm{pH} 2.17$ buffer solution (black) and a basic $\mathrm{pH} 8.05$ buffer solution (red). (a) Fiber probe without hydrogel; (b) Fiber probe with hydrogel. (c) Difference spectra from plasmonic fiber probe referenced to $\mathrm{t}=0 \mathrm{~s}$, after which the analyte solution is cycled between $\mathrm{pH} 2.06$ and 8.05.

The full spectra shift is more evident than the dip shift of the SPR spectra, so the integrated spectra difference can be used to acquire spectra response with higher sensitivity. Summing the absolute magnitudes of the difference spectra over all wavelengths yields an integrated plasmonic response that directly correlates with $\mathrm{pH}$-dependent changes in the properties of the HEMA hydrogel. The $\mathrm{pH}$ response range is experimentally determined using the integrated plasmonic response of fiber probe with a series of increasingly $\mathrm{pH}$ solutions. Each solution is allowed to equilibrate for $10 \mathrm{~min}$, resulting in a quantized integrated response with each step corresponding to a new $\mathrm{pH}$ value. The spectral response is limited by the kinetics associated with the hydrogel swelling/shrinking and, as such, requires equilibrium to be reached in order to make accurate measurements. The integrated spectral response in equilibrium state for each step plotted 
against the $\mathrm{pH}$ value of the solution yields a titration curve in Figure 5. The measurement demonstrates this HEMA modified fiber probe can detect $\mathrm{pH}$ values between 2 to 8 , with the highest resolution when the $\mathrm{pH}$ value is around 5 . This fiber-optics $\mathrm{pH}$ probe covers large $\mathrm{pH}$ range in acid solutions, and its details of response time and $\mathrm{pH}$ resolution will be investigated in future work.

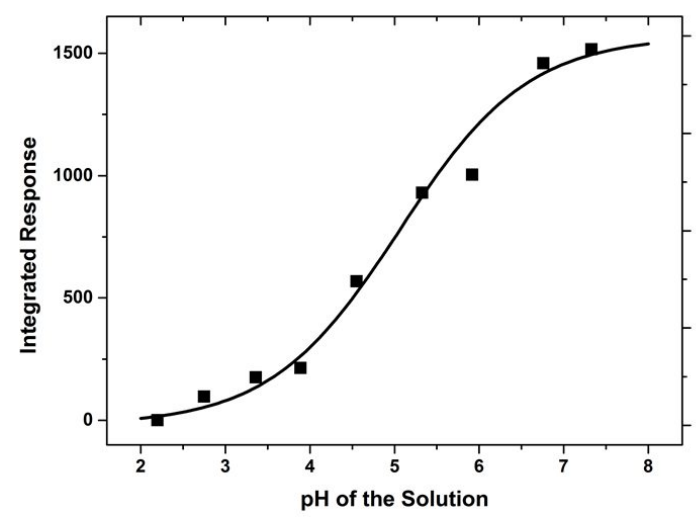

Figure 5. Response of the integrated reflection (black squares) obtained from the measured reflection spectra. The solid line is the titration curves fitted to the experimental data.

\section{CONCLUSION}

In this paper, an arrayed optical fiber facet is obtained by UV-NIL on a gold coating polymer mold. We apply dip coating method to integrate $\mathrm{pH}$-stimuli HEMA hydrogel on this arrayed fiber facet. The plasmonic fiber-optics probe demonstrates $\mathrm{pH}$ sensing capacity in aqueous acid solutions with the highest sensitivity at $\mathrm{pH}$ around 5 . The functional area of this fiber-optic $\mathrm{pH}$ probe is quite miniature and confined on $200 \mu \mathrm{m}$ diameter fiber facet. Besides, the cycling test proves the repeatability of this $\mathrm{pH}$ sensor. The hydrogel integrated fiber probe shows great potential to be a portable, real-time testing, low-cost and robust $\mathrm{pH}$ sensor in biomedical applications.

\section{ACKNOWLEDGEMENTS}

This work was partially supported by the National Natural Science Foundation of China (Grant No. 61306123), the Early Career Scheme of the Research Grants Council of the Hong Kong Special Administrative Region (Award No. 27205515), the Basic Research Program-General Program (JCYJ20140903112959959) from the Science and Technology Innovation Commission of Shenzhen Municipality. The authors thank Xinyu Wang for help on material deposition facility.

\section{REFERENCES}

[1] A. G. Brolo, "Plasmonics for future biosensors," Nat Photon, 6(11), 709-713 (2012).

[2] M. Li, S. K. Cushing, and N. Wu, "Plasmon-enhanced optical sensors: a review," Analyst, 140(2), 386-406 (2015).

[3] C. Wang, and C. Yu, "Detection of chemical pollutants in water using gold nanoparticles as sensors: a review," Reviews in Analytical Chemistry, 32(1), 1-14 (2013).

[4] J. M. Bingham, J. N. Anker, L. E. Kreno, and R. P. Van Duyne, "Gas sensing with high-resolution localized surface plasmon resonance spectroscopy," Journal of the American Chemical Society, 132(49), 17358-17359 (2010).

[5] Y. Shen, J. Zhou, T. Liu, Y. Tao, R. Jiang, M. Liu, G. Xiao, J. Zhu, Z.-K. Zhou, and X. Wang, "Plasmonic gold mushroom arrays with refractive index sensing figures of merit approaching the theoretical limit," Nature communications, 4, (2013). 
[6] J.-F. Masson, "Surface plasmon resonance clinical biosensors for medical diagnostics," ACS Sensors, 2(1), 1630 (2017).

[7] X.-d. Wang, and O. S. Wolfbeis, "Fiber-optic chemical sensors and biosensors (2013-2015)," Analytical chemistry, 88(1), 203-227 (2015).

[8] C. Wang, P. R. Ohodnicki, X. Su, M. Keller, T. D. Brown, and J. P. Baltrus, "Novel silica surface charge density mediated control of the optical properties of embedded optically active materials and its application for fiber optic pH sensing at elevated temperatures," Nanoscale, 7(6), 2527-2535 (2015).

[9] B. Schyrr, S. Pasche, E. Scolan, R. Ischer, D. Ferrario, J.-A. Porchet, and G. Voirin, "Development of a polymer optical fiber $\mathrm{pH}$ sensor for on-body monitoring application," Sensors and Actuators B: Chemical, 194, 238-248 (2014).

[10] C. C. Chiang, C. H. Hsu, and C. H. Ou, "pH Value Detection with CLPFG Sensor." 284, 2157-2161.

[11] S. K. Mishra, and B. D. Gupta, "Surface plasmon resonance based fiber optic pH sensor utilizing Ag/ITO/Al/hydrogel layers," Analyst, 138(9), 2640-2646 (2013).

[12] G. Kostovski, U. Chinnasamy, S. Jayawardhana, P. R. Stoddart, and A. Mitchell, "Sub - 15nm Optical Fiber Nanoimprint Lithography: A Parallel, Self - aligned and Portable Approach,” Advanced Materials, 23(4), 531535 (2011).

[13] F. Baldini, F. Chiavaioli, F. Cosi, A. Giannetti, S. Tombelli, and C. Trono, "Miniaturised optical fiber pH sensor for gastro-esophageal applications." 87941Q-87941Q-4.

[14] E. M. White, J. Yatvin, J. B. Grubbs, J. A. Bilbrey, and J. Locklin, “Advances in smart materials: Stimuli responsive hydrogel thin films,” Journal of Polymer Science Part B: Polymer Physics, 51(14), 1084-1099 (2013).

[15] N. H. Mack, J. W. Wackerly, V. Malyarchuk, J. A. Rogers, J. S. Moore, and R. G. Nuzzo, "Optical transduction of chemical forces," Nano letters, 7(3), 733-737 (2007). 